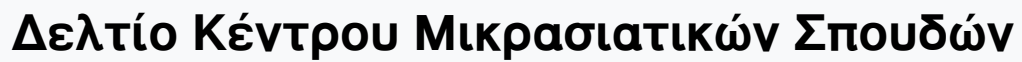

Tóp. 15 (2008)

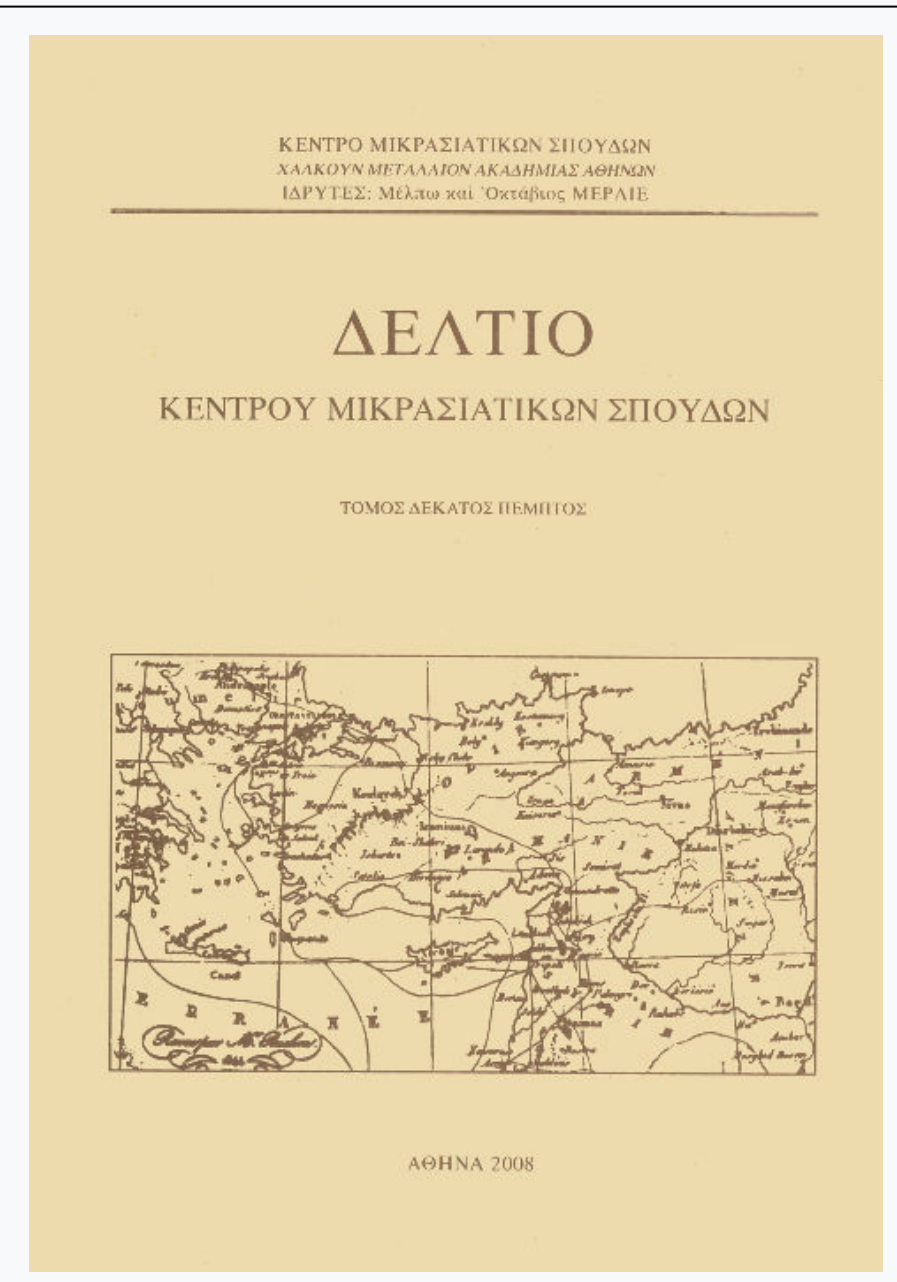

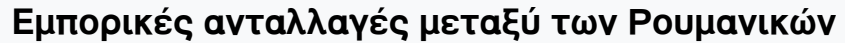

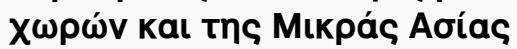

Olga Cicanci

doi: $10.12681 /$ deltiokms.262

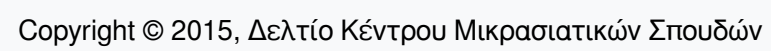

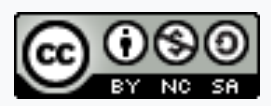

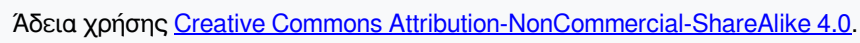

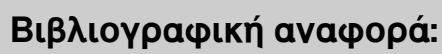

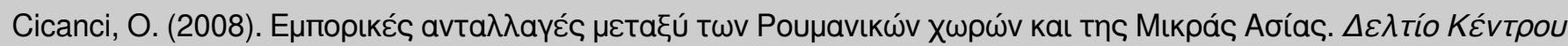

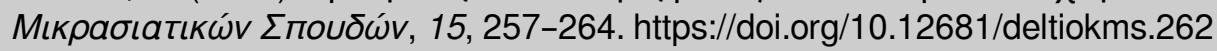




\section{ÉCHANGES COMMERCIAUX ENTRE \\ LES PAYS ROUMAINS ET L'ASIE MINEURE INTERMÉDIÉS PAR LES MARCHANDS GRECS (XVIIe-XIXe SIÈCLES)}

L'annexion par l'Empire ottoman de la majeure partie des régions grecques, suivie au XVIe siècle par l'entrée de la Moldavie et de la Valachie puis de la Transylvanie dans son système politique, aura parmi autres conséquences celle de multiplier les présences grecques dans l'espace roumain. Au XVIIe siècle, lorsque les Grecs constantinopolitains entreprendront leur montée vers les sommets de la hiérarchie administrative de la Porte, leurs contacts avec les Pays roumains deviendront plus fréquents, attirés qu'ils étaient par les avantages économiques présentés par les-dits pays, ainsi que par les chances d'une promotion sociale.

Les marchands représentaient la catégorie sociale la plus nombreuse et la plus active de la diaspora grecque des trois principautés roumaines. Certains de ces marchands ne faisaient que transiter l'espace roumain, mais il y avaient d'autres qui s'y fixaient pour une période limitée ou à demeure, ouvrant des boutiques pour la vente des produits qu'ils se procuraient principalement sur les marchés orientaux, mais aussi en Pologne, Russie, Italie (notamment à Venise) et en Europe centrale. Enrichis, quelques-uns de ces marchands grecs achètent des maisons et des domaines, surtout en Valachie et Moldavie, finissant par s'intégrer définitivement à la vie économique et socio-politique de ces pays, jusqu'à occuper diverses fonctions administratives importantes.

Notons que ces marchands grecs étaient originaires principalement des grands centres économiques de l'Empire ottoman (Constantinople, Trébizonde, Ioannina, Cozani, Mélénic, Philippopolis, Tyrnovo, Arvani- 
tohori, par exemple), mais aussi de Chypre, Chios, Crète et des îles Ioniennes (surtout à partir de la fin du XVIIIe siècle), ainsi que de Venise, Trieste, etc.' Il s'agissait là de relais importants sur les grandes routes marchandes.

Généralement, les marchands grecs, de même que leurs congénères balkaniques (bulgares, serbes, roumains), laissaient sur place des associés aptes à les aider dans leur négoce de transit entre l'Orient et l'Occident, sur mer sans doute, cependant plus encore sur terre ferme. De telles associations (sindrofia) figuraient en tant que telles aussi dans les deux compagnies commerciales grecques de Sibiu (fondée en 1636) et de Braşov (fondée en 1678), villes de Transylvanie méridionale. À part leurs membres grecs constituant la majorité, ces compagnies comptaient aussi des Roumains, des Bulgares, des Serbes, voire des Arméniens et des Juifs, vers la fin du XVIIe siècle à Braşov ${ }^{2}$.

Les noms de quelques-uns des membres des deux compagnies transylvaines se retrouvent également dans les autres pays roumains. Ceux qui se sont fixés à demeure dans l'espace roumain se sont insérés dans la vie économique des pays respectifs, accédant au même statut que les marchands roumains, tout en gardant leurs liens avec leur endroit d'origine, où vivaient d' autres parents ou un associé ${ }^{3}$.

Au XVIIIe siècle, l'espace roumain connaît les maisons de commerce. Dans la plupart des cas, celles-ci se sont épanouies depuis les anciennes sindrofies ou associations marchandes, avec lesquelles elles ont coexisté, du reste.

Les catalogues des documents grecs de Sibiu et de Braşov dressés par Dimitrie Limona ${ }^{4}$, tout comme les deux volumes en collaboration avec Natalia Trandafirescu ${ }^{5}$ qui se rapportent à l'archive de la Maison de com-

1. O. Cicanci, «La spécificité de la diaspora grecque dans l'espace roumain à l'époque moderne», Proceedings of the First International Congress on the Hellenic Diaspora, vol. II, Amsterdam 1991, pp. 105-114.

2. O. Cicanci, Companiile greceşti din Transilvania si comerţul european în anii 1636-1746, Bucarest 1982, p. 96 et suiv.

3. Ibidem, p. 110.

4. Cf. par exemple «Catalogul documentelor referitoare la viata economică în Tările Române în sec. XVII-XIX», Archives d'état de Sibiu, vol. I, Bucarest 1966.

5. Documente economice din arhiva casei comerciale Ioan St. Stamu (17141876), vol. I (1983), vol. II (1988). 
merce Ioan St. Stamu, offrent des données parfaitement claires quant au commerce développé dans les Pays roumains aux XVIIIe-XIXe siècles. À ceci s'ajoutent les études des fonds d'archives inédits effectuées par les étudiants de la Faculté d'archivistes comme ouvrage de diplôme de licence ou de mastérat. Pour le moment, du moins, ces ouvrages (réalisés dans l'intervalle 1996-1999), à quelques rares exceptions près, sont encore inédits; je me propose d'y revenir sur le parcours de cet exposé.

Originaires dans leur majeure partie de l'Empire ottoman, les marchands grecs entrés en relations avec les Pays roumains, en compagnie d'autres congénères de la région balkanique ont tenu un rôle exceptionnel dans le trafic des marchandises de et vers l'Asie Mineure. Ce rôle est mis en lumière par un nombre d'études dont quelques-unes déjà anciennes, dues à Nicolae Iorga ${ }^{6}$, Ion Moga ${ }^{7}$, Andrei Otetea ${ }^{8}$, et d'autres plus rapprochées de nous, celles de M. Dan et S. Goldenberg ${ }^{9}$, Cornelia Papacostea-Danielopolou ${ }^{10}$, Despina Tsourkas-Papasthathis ", etc., ainsi que mes propres contributions ${ }^{12}$, certaines déjà publiées, d'autres attendant encore leur édition, toutes fondées pour une bonne part sur des données nouvelles, inédites.

C'est ainsi que l'on peut constater que si sur la fin du XVIe siècle et au commencement du XVIIe les registres de la douane de Sibiu comportent un

6. Parmi lesquelles, citons: Studii si documente privitoare la Istoria României, Bucarest 1906; Acte româneşti si câteva greceşti din archiva companiei de comert oriental din Braşov, Vălenii de Munte 1932; Points de vue sur l'histoire du commerce de l'Orient à l'époque moderne, Paris 1925, etc.

7. «Politica economică austriacă si comerţul Transilvaniei în veacul al XVIIIlea», Anuarul Institutului de Istorie Natională Cluj, vol. VIII, 1936-19, pp. 86-165.

8. Pătrunderea comerţului românesc în circuitul internaţional, Bucarest 1977.

9. «Le commerce balkano-levantin de la Transylvanie au cours de la seconde moitié du XVIe siècle et au début du XVIIe siècle», RESEE V/1-2 (1967).

10. Comunităţile greceşti din România în secolul al XIX-lea, Bucarest 1996, p.

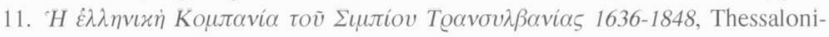
que 1994.

12. À part les travaux précités, voir encore: «Formes d'organisation de l'activité commerciale dans le Sud-Est de l'Europe aux XVIIe et XVIIIe siècles jusqu'au commencement du XIXe siècle», Actes du IVe Colloque international d'Histoire, Athènes 1985, pp. 77-90; «Un Codex inédit de la Compagnie de Sibiu», Revue Roumaine d'Histoire XX (1961), pp. 673-687; «Companiile greceşti din Transilvania: obligaţii fiscale», Studii de istorie economică şi istoria gândirii economice, vol. III, Bucarest 1996, pp. 179-188. 
nombre plutôt réduit de marchandises véhiculées depuis l'Asie Mineure et surtout depuis Trébizonde et Constantinople vers le centre de l'Europe, avec la fondation de la Compagnie commerciale de Sibiu d'abord et de celle de Braşov ensuite, la fréquence du transit augmente sensiblement. La Compagnie de Braşov comptait aussi des marchands originaires de Constantinople, à Braşov, outre les Constantinopolitains, il y avait aussi des marchands de Trébizonde et de Sinope.

Ces marchandises micrasiatiques transitant par Braşov et Sibiu offraient un évantail de produits, à savoir: ceintures, passements, tapis, soieries (notamment de Brousse), boucles, agrafes, fermoirs, mules, babouches, diverses épices, etc. En contre-partie étaient véhiculées sur les routes de Transylvanie et de Valachie des marchandises achetées surtout en Allemagne, Autriche, Bohême et Pologne. Les tableaux que nous avons dressés ${ }^{13}$ des marchandises transitées par les soins des membres des-dites compagnies sont éloquents lorsqu'il s'agit d'évaluer le trafic entre l'Asie Mineure et l'Europe centrale à travers la Valachie et la Transylvanie. Notons à ce sujet qu'une partie au moins de ces marchandises étaient commercialisées sous régime différent dans l'un ou l'autre des pays de l'espace roumain vu les règlements différents qui les régissaient.

Bucarest, la capitale valaque, recevait à l'époque des marchandises venues d'une part de Brousse et d'autre part de Constantinople, ainsi que l'attestent des documents d'archives d'un grand négociant de Braşov, Sigouli Stratou, dont l'activité se déroulait en collaboration avec Manicati Safranou, Iordaki Nicolaou, Polisaki Dimitriou et quelques autres encore. Ces mêmes archives attestent aussi un échange de billets à ordre dans l'intervalle $1694-1697^{14}$.

Les marchands grecs et roumains membres des deux compagnies transylvaines s'approvisionnaient en marchandises dites «turques» sans négliger toutefois les produits valaques et moldaves qu'ils importaient en Transylvanie. Pour ce qui est des marchandises dites «occidentales», ils se les procuraient à Leipzig, Presbourg (Bratislava en tchèque) et Vienne, les vendant ensuite dans les trois principautés roumaines ou les véhiculant dans l'Empire ottoman ${ }^{15}$.

13. O. Cicanci, Companiile greceşti..., p. 176 et suiv.

14. Ibidem, p. 124 et suiv.

15. Ibidem, p. 148 et suiv. 
Après 1742, du fait que l'impératrice Marie-Thérèse concéda par un diplôme aux marchands grecs sujets ottomans le droit de se faire naturaliser autrichiens et de s'établir dans l'Empire autrichien, donc en Transylvanie aussi, le nombre des membres des compagnies de Sibiu et de Braşov devait décliner sensiblement.

Quelques-uns des anciens membres de ces compagnies fondèrent leurs propre maison de commerce, comme le montrent les études de Cornelia Papacostea-Danielopolou, concernant la Compagnie de Braşov, et celles de Oana Barbalata sur la Compagnie de Sibiu pendant la période 1746-1821. La thèse de licence de cette-dernière, intitulée «La Compagnie Commerciale de Sibiu 1746-1821» (inédite jusqu'à présent) repose sur la recherche des fonds conservés aux Archives Nationales de Bucarest et de Sibiu. Oana Barbalata constate grâce à cette recherche que sur la fin du XVIIIe siècle la plupart des marchandises commercialisées par les membres de la Compagnie de Sibiu venaient de Venise, Trieste et Vienne, cependant que les produits fournis par l'Empire ottoman étaient importés par les marchands grecs de Trieste qui les passaient, à leur tour, aux compagnons transylvains. L'un des membres marquants de la Compagnie de Sibiu, Manicati Safranou, dont j'ai déjà mentionné le nom et qui devait par la suite fonder sa propre maison de commerce, trafiquait des billets à ordre et procédait à des échanges de devises à Constantinople avec le concours de Hadji Théodori Dimitriou qui habitait la capitale ottomane.

Il faut convenir que pour le moment il est encore difficile de préciser la date de fondation de telle ou telle maison de commerce par un marchand grec. En effet, si dans le Sud-Est et le Centre de l'Europe existait un réseau de maisons de commerce, leurs archives ne sont pas encore complètement dépouillées et étudiées malgré les progrès en ce sens réalisés jusqu'à présent. On peut quand même mentionner quelques-unes. Notons entre autres la maison de commerce de la famille Stamou de Metzovo avec le siège à Bucarest, de 1714 à 1876, et ses représentants à Iassu, Constantinople, Serres, Vienne, Moscou, Ioannina et, sans doute, à Metzovo même. Une étude récemment achevée et restée en manuscrit réalisée par Mihaela Ciobănescu porte sur «L'évolution de la monnaie et des prix pendant la première moitié du XVIIIe siècle. Documents inédits du fonds Ioan St. Stamou». L'étude examine le jeu des prix dans le commerce des céréales produites par les Pays roumains et vendues sur le marché constantinopolitain, la manière dont le riche marchand respectif prospectait le marché de la capitale ottomane, l'activité commerciale dévelopée par les ports de Braìla 
et Galatzi, etc. Une autre maison commerciale ayant tenu un grand rôle dans le commerce international des Pays roumains fut la Maison Polizou, dont j'ai eu déjà l'occasion de parler ${ }^{16}$. Il convient toutefois de mentionner qu'elle comptait parmi ses collaborateurs Constantin Darvaris et son frère siégeant à Zémun et Vienne et Bucarest mais qui se fournissaient en produits orientaux à Constantinople pour les vendre ensuite en Europe centrale. De leur côté, Dimitrie Engürlui et Vasile Iconomu, douanier à Galatzi, achetaient des marchandises à Constantinople et s'en défaisaient en Valachie ${ }^{17}$.

En dépouillant l'Archive Nationale de Galatzi, Valentina Filip a étudié 169 documents rédigés en grec. Il s'agissait, dans la plupart des cas, de la correspondance des frères Kiritza et Dimitrie Engürlui échangée avec des marchands de Sibiu ${ }^{18}$ et de Braşov, dans l'intervalle des années 1811-1832. Les deux frères étaient originaires de Constantinople où ils étaient représentés par le père de Hadji Théodori Dimitriou précité et s'occupaient de l'importation du coton, de la soie, de la laine, des peaux, des épices et autres produits, qu'ils vendaient dans les Principautés roumaines ou bien véhiculaient vers l'Europe centrale par la route de Transylvanie.

Les mêmes archives de Galatzi comportent environ 80 documents «karamanlis». Ils sont étudiés (au point de vue historique et linguistique) par le jeune chercheur Claudiu Turcitu, dans sa thèse de licence et mastérat, intitulée «Les Pays Roumains dans le commerce entre l'Orient et l'Europe centrale au commencement du XIXe siècle» ${ }^{19}$.

Ces documents se rapportent également à l'activité des frères Engürlii qui, établis à Braşov et à Bucarest exerçaient le commerce des marchandises orientales, en collaboration avec des Roumains et des Grecs. Retenons l'intérêt des données concernant les marchandises dont bon nombre étaient importées de l'Empire ottoman, où les deux frères avaient leurs

16. O. Cicanci, «L'activité commerciale de Kiriaki Polizou dans les Principautés roumaines vers la fin de l'époque phanariote d'après les archives de l'église grecque de Vienne», Symposium «L'époque phanariote», Thessalonique 1974, pp. 26-411.

17. O. Cicanci, «Rolul Casei de Negot Polizu în comertul international al Tarilor Române, Sud-Estul şi contextul european», Bullein I (Bucarest 1994), pp. 129-140.

18. Cf. la thèse de diplôme (ms.) intitulée «Tările Române în comertul dintre Orient şi Europa Centrală la începutul secolului al XIX-lea».

19. Thèse de licence et mastérat intitulée «Documente karamanice dion Arhivele Naţionale ale României». 
familles (à Constantinople), et les prix de vente des marchandises respectives, défaites ou transitées dans les Pays roumains.

Par le moyen des centres marchands de Constantinople et d'Asie Mineure, les Principautés roumaines étaient approvisionnées aussi en marchandises occidentales, françaises et anglaises entre autres, ainsi qu'il résulte pour la première moitié du XIXe siècle des travaux de recherches de Anca Dobre et Felicia Diaconu ${ }^{20}$. À titre d'exemple, je mentionne le cas de la firme des frères Théologou de Constantinople, qui achetait à Marseille, Lyon, Londres, Manchester, ailleurs aussi, des produits fournis par les firmes grecques des centres respectifs, transportés ensuite jusqu'aux ports roumains de Galatzi et Braila par des navires grecs battant pavillon anglais.

Malgré leur intérêt, il n'y a pas lieu ici de s'appesantir sur les données attestant la présence des marchands grecs en tant qu'intermédiaires entre l'Asie Mineure et l'espace roumain, données mises au jour par les études des jeunes chercheurs sortis de l'École des archives bucarestoise. De ces études, de même que de mes propres recherches se dégage la portée du rôle tenu par les marchands grecs dans le développement nourri en premier lieu par les échanges avec l'Asie Mineure.

Pour commencer, vers la fin du XVIe siècle et le début du XVIIe les marchands grecs s'occupaient surtout de l'achat des produits que les Pays roumains étaient tenus à fournir à la Porte, suite de leur statut politique. Aux siècles suivants, ces mêmes marchands réunis en associations, familiales dans la plupart des cas, en compagnies commerciales et maisons de commerce, deviendront les principaux trafiquants des marchandises orientales, micrasiatiques tout d'abord, dans l'espace roumain. Mais ce trafic s'exerçait aussi selon un incessant va-et-vient vers et de l'Europe centrale. En fin de compte, quelques-uns de ces marchands se sont fixés à demeure dans les Pays roumains, devenant des propriétaires fonciers sans renoncer à leur activité commerciale, mais s'intégrant dans la vie socio-politique et culturelle de leur pays d'adoption.

Depuis le milieu du XVIIe siècle et au commencement du XIXe siècle, les traités conclus par la Porte avec les puissances occidentales favorisèrent

20. Un aperçu des thèses de licence dans l'article intitulé: «Rolul fraţilor Gheorghiev în comerţul franco-englez al Tărilor Române», Buletin al Facultăţii de Archivistică, IIm Bucarest 1996, pp. 211-215. 
les marchands grecs, de même que les marchands roumains et balkaniques en général, dont les uns étaient sujets ottomans, mais les autres étaient naturalisés autrichiens, prussiens, anglais ou russes. Cette situation conduisit à un véritable réseau de maisons de commerce et de firmes qui couvrait l'ensemble du Sud-Est européen, avec une priorité marquée pour l'activité portant sur l'espace micrasiatique, roumain et central européen. 